\title{
Enhancement and summation in the perception of two successive vibrotactile stimuli
}

\author{
RONALD T. VERRILLO \\ Institute for Sensory Research, Syracuse University, Merrill Lane, Syracuse, New York 19210 \\ and
}

GEORGE A. GESCHEIDER

Hamilton College, Clinton, New York 13923

\begin{abstract}
Enhancement and summation were found to be fundamentally different perceptual processes affecting the sensation magnitude of two successive vibrotactile stimuli. Enhancement, defined operationally as an increment in the subjective magnitude of one stimulus due to the presentation of a prior stimulus, and summation, defined as an increment in overall subjective magnitude of the two stimuli, were measured for sinusoidal vibration of the thenar eminence of the hand. The effect of summation was maximum when the two stimuli greatly differed in frequency, whereas maximum enhancement effects were found when both stimuli were close in frequency. The summation effect showed little decay as the interstimulus interval was increased to as much as $500 \mathrm{msec}$, whereas enhancement effects decayed to zero at approximately 500 msec. Results were similar to those obtained in comparable studies of audition and support the hypothesis that there are at least two distinct information-processing channels for the perception of cutaneous vibration.
\end{abstract}

The results of experiments by Zwislocki and his associates clearly indicate that enhancement and summation are fundamentally different processes which determine auditory sensation magnitude in the perception of pairs of consecutively presented brief sound bursts (Cannon \& Zwislocki, 1970; Irwin \& Zwislocki. 1971; Zwislocki \& Ketkar, 1972; Zwislocki. Ketkar. Cannon, \& Nodar, 1974; Zwislocki \& Sokolich. 1974). Loudness enhancement was defined operationally as the loudness-level increment of a sound burst due to the presentation of a prior burst, while loudness summation referred to an increment in the overall loudness level of the pair. Scharf (1969) has made a distinction between analogous processes as they affect loudness perception of dichotically presented, simultaneous tone bursts having different frequencies. According to Zwislocki et al. (1974). disagreement among the results from studies of temporal summation of suprathreshold stimuli may be due to varying degrees of uncontrolled interaction between the two perceptual processes.

Zwislocki et al. (1974) measured loudness enhancement and summation by employing a loudness matching procedure in which a third burst following the burst pair was adjusted for loudness

The material of this article was presented at the 1974 meeting of the Psychonomic Society. The research was supported by Grant NS-04940 from the National Institutes of Health. U.S. Department of Health. Education and Welfare. The authors are grateful to Dr. Jozef Zwislocki for his interest and help throughout all phases of the research. comparison. A loudness enhancement measurement was made by instructing the subject to match the loudness of the comparison burst to that of the second burst. Loudness summation was measured by instructing the subject to match the loudness of the comparison burst to the total loudness of the burst pair.

It was found that subjects were not consistently able to make the kind of judgment prescribed by the experimenter's instructions. For example. subjectiveintensity judgments of the second stimulus were more easily made when the frequency of the second stimulus in the pair was different from that of the first stimulus but the same as that of the comparison stimulus. When the frequency of the comparison stimulus differed from either the first or second stimulus of the pair and the time interval between the stimuli in the pair was short. subjects often matched the comparison stimulus to the total loudness of the pair rather than to the loudness of the second stimulus. Under these conditions, the contaminated judgments may reflect loudness summation rather than loudness enhancement. Similarly. judgments of the total loudness of the pair may be contaminated by the subject's tendency to judge only the second stimulus under conditions in which it is difficult to attend to the overall loudness of the burst pair. $\mathrm{Zw}$ islocki and his associates found that this error tendency was particularly strong when the second burst of the pair was perceptually similar to the comparison burst and when the time interval between 
the stimuli of the pair was relatively long. In general, subjects tend to match the loudness of the comparison burst to the loudness of that portion of the stimulus pair which perceptually most resembles the comparison burst. This generalization has been called the "principle of maximum similarity" (Zwislocki \& Ketkar, 1972).

The present study was designed to investigate enhancement and summation phenomena in the perception of a pair of temporally spaced, brief vibrotactile stimuli. The first experiment was an attempt to test the applicability of the principle of maximum similarity in the cutaneous modality. In subsequent experiments, variables that had been investigated in the auditory studies (stimulus frequency and the intensity and time difference between the two stimuli of a pair) were manipulated to determine whether common principles could account for enhancement and summation effects in both the auditory and cutaneous modalities.

\section{METHOD}

In all experiments, data were obtained from either five or six well-trained subjects. Training consisted of several sessions in which the subjects made enhancement and summation judgments for pairs of stimuli separated by various time intervals.

Sinusoidal vibratory stimuli were delivered to the thenar eminence of the subject's hand. The $2.9-\mathrm{cm}^{2}$ contactor of the vibrator was adjusted at the start of each experimental session to make a $0.5-\mathrm{mm}$ depression in the skin surface under the contactor. Vibration of the skin was restricted to the region of the contactor by using a rigid surround upon which the subject rested his hand. Between the surround and the contactor was a $1.0-\mathrm{mm}$ gap. Vibration amplitude was measured with a calibrated accelerometer mounted on the moving element of the vibrator, and all measurements were expressed in $\mathrm{dB}$ re 1.0 micron peak displacement of the contactor. Stimuli were $20 \mathrm{msec}$ in duration at half-power points with a $25-\mathrm{msec}$ rise/fall time and had approximately Gaussian envelopes. In all experiments, the second stimulus in the pair had a sensation level of $24 \mathrm{~dB}$.

The subject and the vibrator assembly were located in a booth to provide isolation from vibrations of the building and sound in the laboratory. The sound of the vibrator was masked by a continuous narrow-band noise centered at the frequency of the vibratory stimuli.

Instructions which produced enhancement effects required the subject to match the sensation magnitude of the comparison stimulus to the sensation magnitude of the second stimulus of the pair while disregarding the first stimulus. Instructions for measuring summation effects required the subject to match the comparison stimulus to the total sensation magnitude of the pair. The comparison stimulus was presented $750 \mathrm{msec}$ after the second stimulus in the pair. The time interval between the onsets of the first and the second stimulus of the pair was manipulated over a range of 35 to $500 \mathrm{msec}$. In each trial, the subject adjusted the intensity of the comparison stimulus by adjusting a smooth unmarked knob attached to a logarithmic, continuously variable attenuator. The subjects were trained to use a bracketing method: on a single trial, they made several adjustments of the knob to the upper and lower limits of a match, after which they adjusted the subjective intensity toward the center of this interval of uncertainty.

In each experiment, the subjects made between two and six matches for each experimental condition. The order of presentation for stimulus pairs of various interstimulus time intervals was randomized within each experimental session. The order of presentation of other variables was counterbalanced either over blocks of trials within a session or over sessions. Within each session, two control matches were made in which the subject matched the comparison stimulus to the second stimulus of the pair in the absence of the first stimulus. Thus, the effects of the first stimulus in the pair could be expressed as the difference between experimental matches involving the pair and control matches involving only the second stimulus of the pair.

At the start of each session, the subject's threshold was measured by the method of limits and the intensity of the second stimulus of the pair was set at a sensation level of $24 \mathrm{~dB}$. A matching procedure was then employed to equate the sensation magnitudes of both stimuli of the pair. The first stimulus and the second stimulus of the pair were accepted by the experimenter as equal in sensation magnitude when the subject's adjustments of the comparison stimulus to each stimulus alone were within $1 \mathrm{~dB}$ or less of each other. In some experiments on enhancement, the first stimulus was reduced or increased $10 \mathrm{~dB}$ relative to the value established by this initial matching procedure.

Statistical analysis of the data consisted of computing the mean and standard deviation at each interstimulus time interval for every experimental condition. The standard deviations were relatively small, ranging from 0.09 to $1.88 \mathrm{~dB}$, with a mean of $0.87 \mathrm{~dB}$. Standard deviations of $1.0 \mathrm{~dB}$ or less accounted for $71 \%$ of the data.

\section{RESULTS AND DISCUSSION}

\section{The Principle of Maximum Similarity}

The initial experiment required subjects to adjust the subjective intensity of the comparison burst under both sets of instructions, using burst frequencies of $100-500-500 \mathrm{~Hz}$ and $100-500-300 \mathrm{~Hz}$. The first two frequencies refer to the stimulus pair and the third to the comparison stimulus. These frequencies were selected in order (1) to maximize the similarity between the comparison stimulus and the second stimulus in the case of the $100-500-500 \mathrm{~Hz}$ combination, (2) to maximize the discriminability (established by prior testing) between the three stimuli for the $100-500-300 \mathrm{~Hz}$ combinations, and (3) to insure that all frequencies were within the range of the high-frequency mechanoreceptors.

The results plotted in Figure 1 are based on the means of the judgments made by five subjects. The average difference in decibels between matches in which both stimuli in the pair were presented and control matches in which only the second stimulus of the pair was presented is plotted as a function of the time interval between the first and second stimulus of the pair. Although the parameter values of the stimulus pair were the same, changing the instructions and the frequency of the comparison stimulus clearly affected the judgment of the subjects.

The smallest effect was observed when the subject was instructed to match the sensation magnitude of the comparison stimulus at the sensation magnitude of the second stimulus of the pair and the vibration frequencies of these stimuli were the same. According to the principle of maximum similarity, matching to 


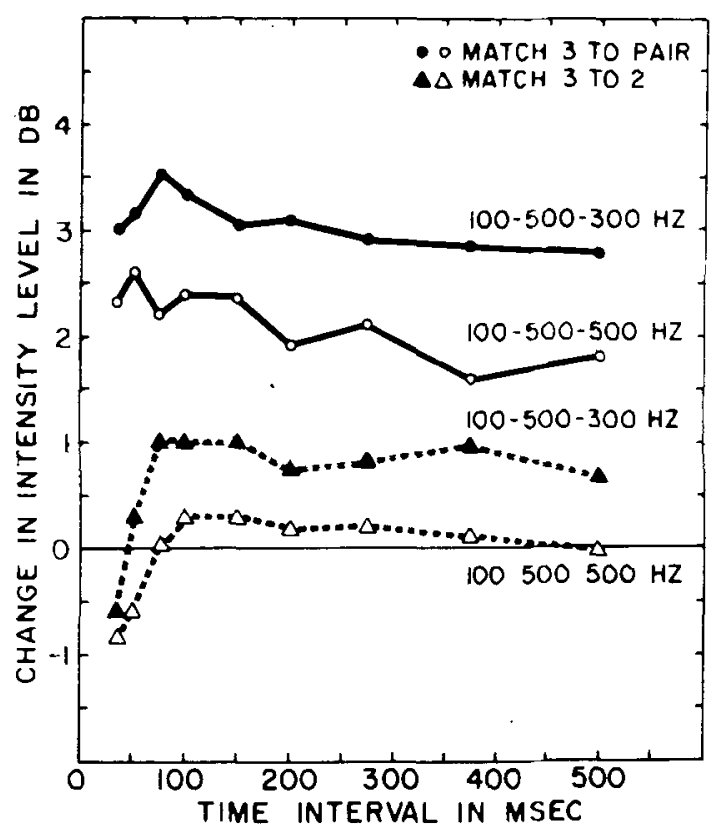

Figure 1. Chenge in the intenity kved of a third atmalus, when matched diher to the orerall subjective magnitude of a preceding stimulas patr (eammation instructions) or to the second stholus in the pair (enhancement instructions), a a function of the time mterval between the stimali of the pair. Vibration frequenctes of the three afinoll are indicated on the graph. Data pointi indicate the means of five sabjects.

the second stimulus of the pair is easiest when the comparison stimulus is perceptually similar to the second stimulus. A preceding stimulus does not appreciably affect the sensation magnitude of a following stimulus when both have been previously equated in sensation magnitude. An equivalent result was found for audition under comparable conditions (Irwin \& Zwislocki. 1971; Zwislocki et al., 1974). In subsequent experiments, this finding was obtained using a variety of different frequency combinations in which the comparison stimulus and the second stimulus of the pair were the same frequency (Figure 2). When the comparison and second stimuli were different, as in the $100-500-300 \mathrm{~Hz}$ combination, there is an approximate $1.0 \mathrm{~dB}$ effect.

The largest effect (Figure 1) occurred when the subjects attempted to match the sensation magnitude of the comparison stimulus to the total sensation magnitude of the stimulus pair and the vibration frequency of the comparison stimulus was the arithmetic mean of the frequencies of the pair. In this situation. the judgments are consistent with the principle of maximum similarity in that the comparison stimulus is perceptually different from either the first or second stimulus of the stimulus pair. Zwislocki et al. (1974) also found the greatest loudness effects under comparable conditions. The effect of instructions to match to the loudness of the pair is relatively independent of the interstimulus time interval up to at least $500 \mathrm{msec}$, an effect which was also observed for auditory stimulation (Zwislocki et al.. 1974). The time independence suggests that the summation process at suprathreshold levels is different from that of temporal summation in detecting threshold stimuli where the time constant is on the order of $200 \mathrm{msec}$ for both auditory (Zwislocki, 1960) and vibrotactile stimulation (Verrillo, 1965).

The intermediate results can be explained by assuming that the subjects had difficulty following the instructions when the task was not consistent with the principle of maximum similarity. With instructions to match the subjective intensity of the pair (Figure 1) when the frequency of the second stimulus and the comparison stimulus were the same $(500 \mathrm{~Hz})$, the subject may have had a tendency to match to only the second stimulus. When asked to match to the second stimulus (Figures 1 and 2) when the comparison stimulus frequency $(300 \mathrm{~Hz})$ was different from that of the second stimulus $(500 \mathrm{~Hz})$ of the pair, the subject may have incorrectly matched to the pair. The difficulty in making judgments of the subjective magnitude of the pair when the comparison stimulus and the second stimulus of the pair have the same frequency was also found by Zwislocki et al. (1974) for audition. The results for the two modalities suggest that data obtained concerning the total subjective magnitude of the pair may be contaminated by tendencies to make judgments of a single stimulus when one of the stimuli of the pair is perceptually similar to the comparison stimulus. When the frequency of the comparison stimulus $(300 \mathrm{~Hz})$ was different from that of the second stimulus $(500 \mathrm{~Hz})$ in the pair, the second stimulus was judged higher than when the two stimuli had the same frequency

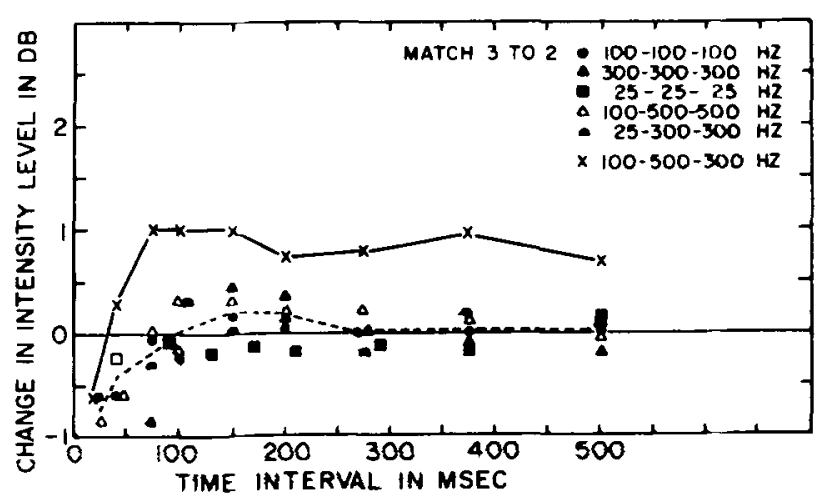

Figure 2. Change in the intensity level of a third stimolus when matched to the subjective magnitude of the second stimplns of a preceding pair (enhancement instructions), as a function of the time interval between the stimali of the pair. A condition $(X)$ in which the comparison stimulus is different in frequency from that of the second stimulus is compared with a variety of conditions in which the comparison and recond stimuli have the same frequency. 
$(500 \mathrm{~Hz})$. This result was also obtained for auditory stimuli and was attributed to a tendency for subjects to incorrectly match the comparison stimulus to the total loudness of the pair rather than to the loudness of the second stimulus of the pair (Zwislocki et al., 1974). It appears that a similar contamination of responses can occur for vibrotactile stimulation.

According to the present results and those obtained in auditory studies, at least two distinct processes must be considered in accounting for sensation magnitude effects in pairs of brief temporally separated stimuli. One process concerns the sensation magnitude of each individual stimulus and has been termed enhancement; the other concerns the total sensation magnitude of the stimulus pair and has been termed summation (Zwislocki et al., 1974). For both auditory and vibrotactile stimuli, the process that is measured depends on the instructions to the subject and on the frequency of the comparison stimulus relative to the component frequencies of the pair. The effect of the frequency of the comparison stimulus demonstrates a tendency for the subject to match the comparison stimulus to that perceptual aspect of the pair which most resembles the perception of the comparison stimulus. It appears that, unless the subject's task is consistent with the principle of maximum similarity, the results may be ambiguous for both auditory and vibrotactile stimuli. Zwislocki et al. (1974) suggest that failure to observe the principle may have led to the inconsistent results obtained in studies of temporal loudness effects. Our findings indicate that similar problems may arise in studying temporal interaction in other modalities when the principle is not taken into account.

\section{Vibrotactile Information-Processing Channels}

The 3-dB effect (Figure 1) obtained in matching the subjective intensity of the pair when the frequency of the comparison stimulus was the mean of the frequencies of the stimuli in the pair is considerably less than the 10-dB effect reported by Zwislocki et al. (1974). They used pairs of auditory tone bursts in which the first and second burst of the pair were 1 and $4 \mathrm{kHz}$, respectively, and the comparison burst was $2 \mathrm{kHz}$. A $10-\mathrm{dB}$ increase in a $2-\mathrm{kHz}$ tone is approximately equivalent to doubling loudness (Stevens, 1955), but an increase of approximately $6 \mathrm{~dB}$ is necessary to double the subjective magnitude of a $300-\mathrm{Hz}$ vibrotactile stimulus (Verrillo, Fraioli, \& Smith, 1969).

According to critical band theory (Zwicker, Flottorp, \& Stevens, 1957; Zwicker \& Scharf, 1965), the loudness of a sound can be calculated by integrating loudness over all critical bands excited by the sound. Within each critical band, acting as a separate channel, the loudness is determined by a nonlinear transformation of the total energy from the frequency components within each band. At some point after the separate nonlinear transformations of energy to loudness, the total loudness of the sound is produced by the summation of the loudnesses generated at each critical band. From this formulation, it follows that equally loud 1 - and $4-\mathrm{kHz}$ tones, because they are in widely separated critical bands, should be judged to be twice as loud as either tone alone. Thus, in the experiments on auditory temporal summation, perfect loudness summation occurred when the subject's adjustment of a comparison tone to match two equally loud tones from different critical bands was $10 \mathrm{~dB}$ higher than, and consequently twice as loud as, his match to either tone alone. On the other hand, presenting two tones of equal energy within the same critical band results in a match of the comparison stimulus which is only approximately $3 \mathrm{~dB}$ higher for the, pair of tones than for one tone alone (Scharf, 1970). The effect decreases as a function of the time interval between the tones. If the critical band formulation for audition is extended to our results with vibrotactile stimuli, we must conclude that $100-$ and $500-\mathrm{Hz}$ stimuli are within the same information-processing channel.

There is considerable evidence that stimuli above and below $40 \mathrm{~Hz}$ are processed by different receptor populations. An information-processing channel for the detection of high-frequency vibratory stimuli such as those used in our first experiment has been identified by both psychophysical and physiological procedures. The psychophysical studies of Verrillo (1966a, b; 1968) in combination with electrophysiological work by Hunt (1961), Loewenstein and Mendelson (1965), Sato (1961), and Talbot, Darian-Smith, Kornhuber, and Mountcastle (1968) clearly indicate that the Pacinian corpuscle is the receptor responsible for the perception of vibration above $40 \mathrm{~Hz}$. Since the Pacinian corpuscle is a velocity-sensitive receptor, it will not easily fire at frequencies below $40 \mathrm{~Hz}$, because the critical velocity can be reached only at extremely high vibration amplitudes at these low frequencies. Below $40 \mathrm{~Hz}$, another system operates for the detection of vibration. The two systems appear to have very different operating characteristics. Verrillo (1968) has found that the high-frequency system produces a U-shaped frequency response curve and exhibits spatial and temporal summation. The low-frequency system produces a flat frequency response and exhibits neither spatial nor temporal summation at threshold levels of stimulation.

The 3-dB effect obtained in the present experiment (Figure 1) indicates that moderately intense vibrotactile stimuli differing in frequency but well within the frequency response range of the Pacinian corpuscle is analogous to results obtained when two auditory tones having frequencies within the same 
critical band are presented to a listener. It is implied from auditory critical band theory that sensation magnitude rather than stimulus energy is summated when stimuli are presented to separate informationprocessing channels. The $10 \mathrm{~dB}$ of auditory temporal summation when stimuli were in different critical bands (Zwislocki et al., 1974). the $3 \mathrm{~dB}$ of summation obtained for stimuli in the same critical band (Scharf, 1970), and the $3 \mathrm{~dB}$ of vibrotactile temporal summation obtained in the present study when stimuli are processed by the same cutaneous receptor population are all consistent with this hypothesis. A further implication of the hypothesis is that sensation magnitude may be summated when vibrotactile stimuli are processed by separate receptor populations. To test this implication, an experiment was conducted in which the first stimulus of the pair was $300 \mathrm{~Hz}$ and the second was $25 \mathrm{~Hz}$.

According to Verrillo's (1968) psychophysical data, it appears that a $25-\mathrm{Hz}$ stimulus at a sensation level of $24 \mathrm{~dB}$ delivered to the hand by a $2.9-\mathrm{cm}^{2}$ contactor should have a relatively small effect on the Pacinian system. Based upon the assumption that the low-frequency system has a flat-frequency response (Verrillo, 1968) and upon the shapes of the equal vibratory sensation contours of Verrillo, Fraioli, and Smith (1969), it appears that a $300-\mathrm{Hz}$ stimulus matched in sensation magnitude to a $25 \cdot \mathrm{Hz}$ stimulus at 24-dB sensation level will not be of sufficient intensity to excite low-frequency receptors. Assuming a relative isolation of the two channels, the prediction can be made that presenting stimuli of equal sensation magnitude to each channel should result in approximately twice the sensation magnitude of either stimulus alone. In our experiment, the comparison stimulus was $80 \mathrm{~Hz}$. It is reasonable to assume from magnitude estimation data (Stevens, 1959; Verrillo et al., 1969) that, for stimuli above the 10- or 15-dB sensation level, the exponent of the psychophysical function for $80 \mathrm{-Hz}$ vibration is approximately 0.5 when stimulus intensity is expressed in energy terms. It therefore follows that a $6-\mathrm{dB}$ increase in stimulus intensity corresponds to a doubling of sensation magnitude.

The results presented in Figure 3, based on the judgments of five subjects, indicate that when the stimuli were 300 and $25 \mathrm{~Hz}$ the effect obtained by instructions to match to the subjective intensity of the pair was as much as $6 \mathrm{~dB}$, but that when the stimuli were 100 and $500 \mathrm{~Hz}$ the effect was reduced to approximately $3 \mathrm{~dB}$. The results shown in Figure 4 are a further confirmation of the observation that two stimuli presented within a single informationprocessing channel $(25-25-25 \mathrm{~Hz}$ or $300-300-300 \mathrm{~Hz})$ produces an effect of approximately $3 \mathrm{~dB}$. These findings strongly support the conclusion that in vibrotaction as well as in audition temporal summation of suprathreshold stimuli is determined by

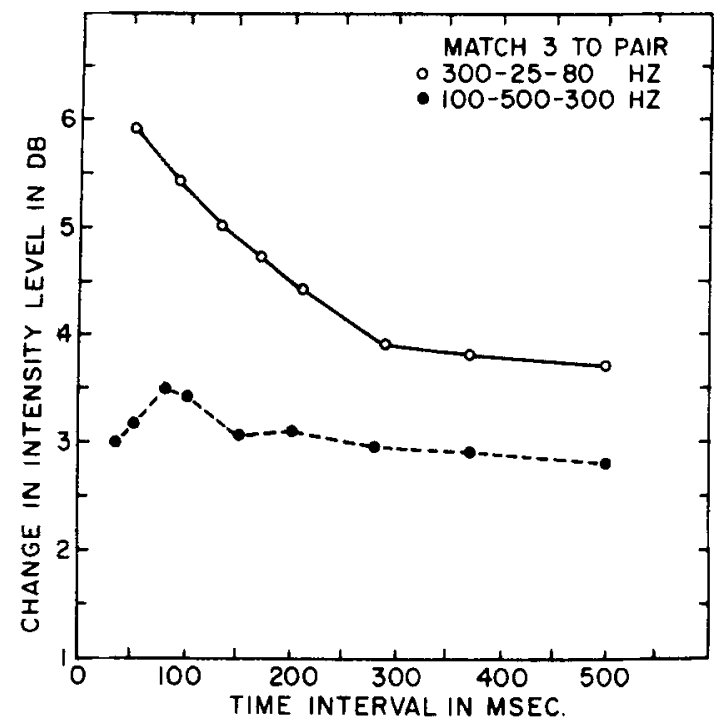

Figure 3. Change in the intensity level of a third stimulus, when matched to the subjective magnitude of a preceding stimulus pair (summation instructions), as a function of the time interval between the stimuli of the pair. Vibration frequencies are indicated on the graph. Data points indicate the means of five subjects.

the integration of sensation magnitudes when stimuli are presented to separate information-processing channels. Furthermore, the results provide additional support for the hypothesis that at least two functionally distinct channels are involved in the perception of mechanical vibration on the skin.

\section{Effects of Frequency and Intensity}

In audition, loudness enhancement was found to be negligible when the two tones had the same loudness level (Zwislocki et al., 1974). This resulted both when the tones were within the same critical band as well as when they were in different critical bands. When the loudness of the first tone was increased, however,

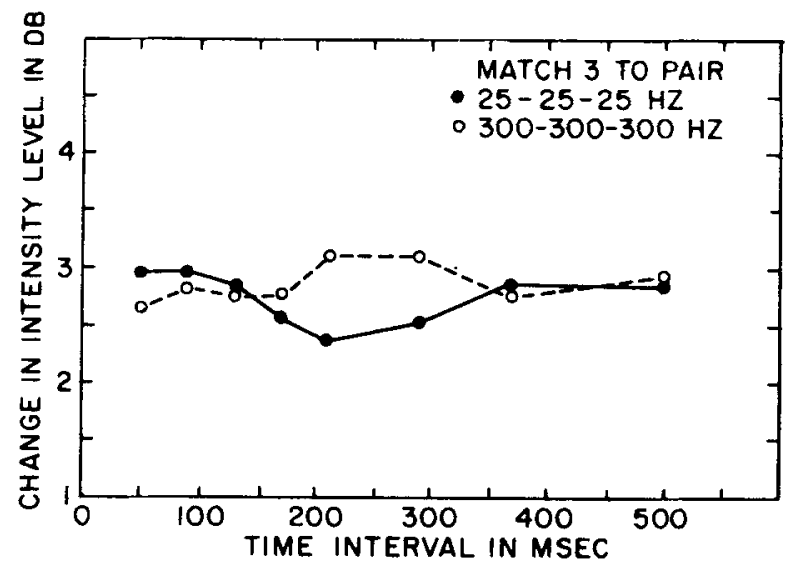

Figure 4. Response to summation instructions plotted as a function of the time interval between the first two stimuli. Comparison between low- and high-frequency cutaneous mechanoreceptor channels. 


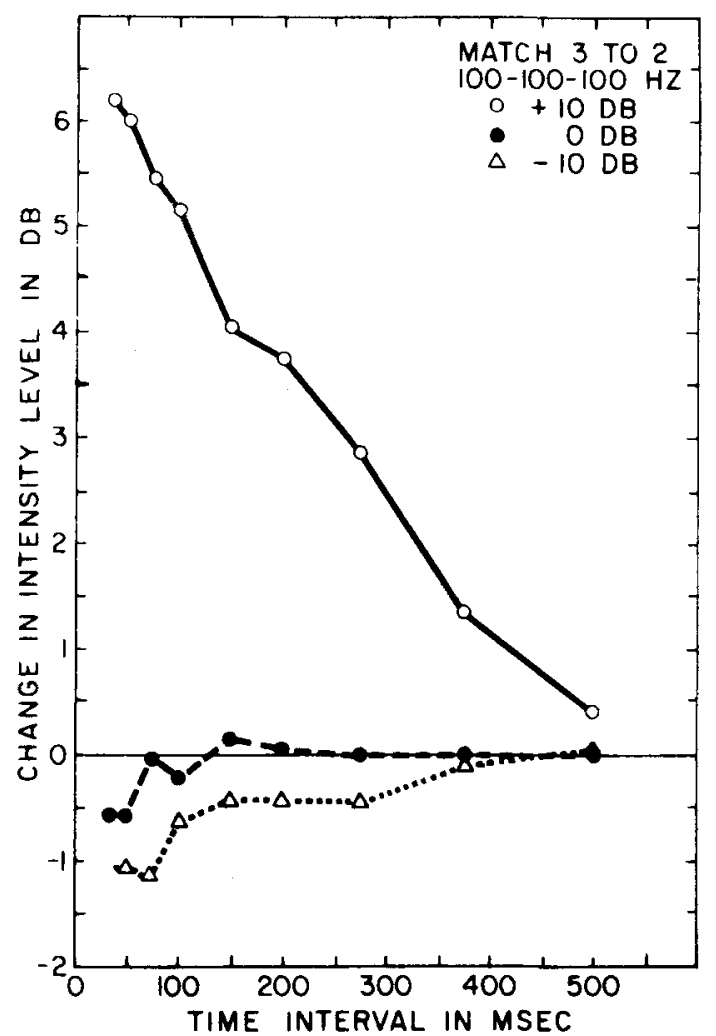

Figure 5. Change in the intensity level of a third stimulus, when matched to the second stimulus of a preceding pair (enhancement instructions), as a function of the time interval between the first two stimuli. The first stimulus in the pair was set at $+10,0$, or $-10 \mathrm{~dB}$ re the intensity of the second stimulus. Mean data obtained from six subjects are shown.

there was a substantial enhancement effect if the two tones were in the same critical band (Zwislocki \& Sokolich, 1974). Variation of the frequency difference between the two tones has been found to have opposite effects for the two sets of instructions. Zwislocki and Sokolich found that loudness enhancement was maximum when the frequencies of the two tones were nearly the same and thus in the same critical band, whereas Scharf (1970) found loudness summation was minimal when the frequencies of the tones were within the same critical band.

A series of experiments was conducted to investigate intensity and frequency parameters, to more precisely differentiate between enhancement and summation phenomena in the cutaneous modality, and to make further comparisons with analogous phenomena in the auditory modality. In the first experiment, both stimuli in the pair had a frequency of $100 \mathrm{~Hz}$ and the comparison stimulus was also $100 \mathrm{~Hz}$. The intensity of the first stimulus was $+10,0$, or $-10 \mathrm{~dB}$ relative to the level of the second stimulus. The subject was instructed to match the comparison stimulus to the second stimulus of the pair. The mean results of six subjects are shown in Figure 5. They are remarkably similar to those obtained by Zwislocki and Sokolich (1974) when the two tones of the pair and the comparison stimulus were $1 \mathrm{kHz}$. Analogous dichotic experiments by Galambos, Bauer, Picton, Squires, and Squires (1972) yielded a similar result. In both modalities, enhancement effects are negligible when the first and second stimulus of the pair are the same intensity but become substantial when the first stimulus is more intense than the second stimulus. In both modalities, when the first stimulus was weaker than the second stimulus, it tended to reduce the sensation magnitude of the second stimulus. The effect of the first stimulus gradually decays within about $500 \mathrm{msec}$. The basic findings of this experiment do not appear to be dependent on stimulus frequency, as evidenced by the results presented in Figure 6, where all three stimuli were $300 \mathrm{~Hz}$, and in Figure 7, where all three stimuli were $25 \mathrm{~Hz}$.

Zwislocki and Sokolich (1974) argued that loudness enhancement functions may be contaminated by tendencies of subjects to make judgments of the total subjective intensity of the pair when the interstimulus time interval was so short that the two stimuli of the

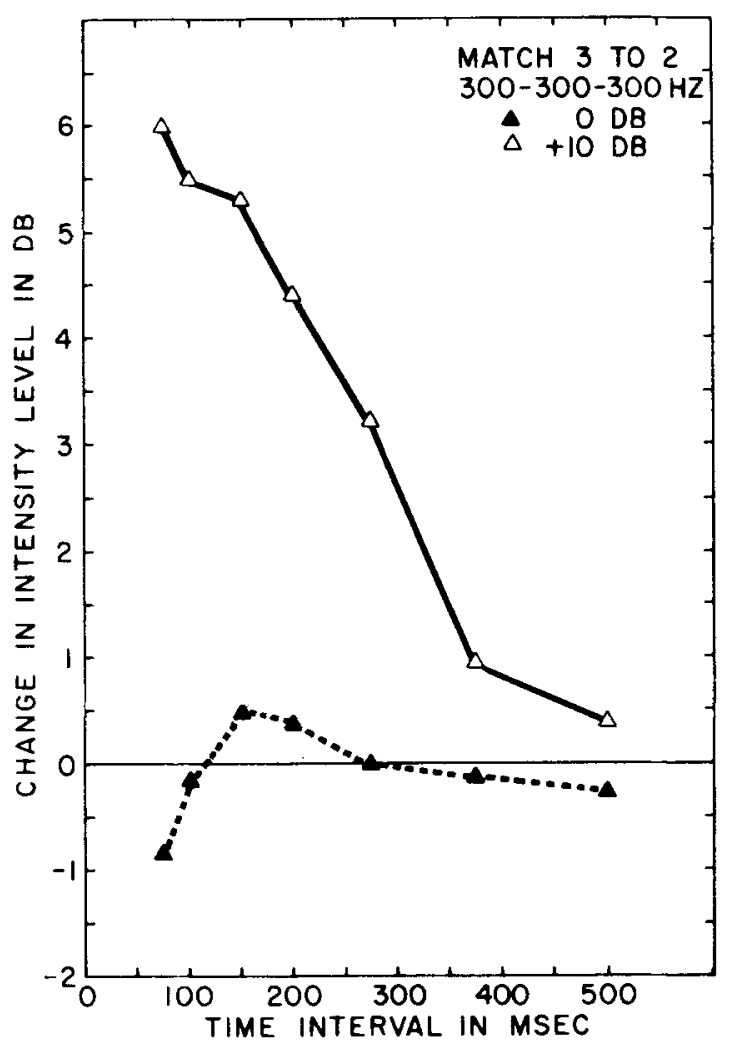

Figure 6. Responses to enhancement instructions plotted as a function of the time interval between the first two stimali. The first stimulus in the pair was set at either 0 or $+10 \mathrm{~dB}$ re the intensity of the second stimulus. The stimulus frequencies $(300-330-300 \mathrm{~Hz}$ ) activate primarily the high-frequency mechanoreceptor system. Mean data of five subjects are shown. 
pair appear fused or nearly fused. The tendency for all three 10-dB curves in Figures 5,6, and 7 to level off at $150 \mathrm{misec}$ and then to rise at shorter interstimulus time intervals suggests that vibrotactile enhancement curves may not reflect the enhancement phenomenon at short time intervals but instead may be contaminated by the tendency of subjects to judge the subjective intensity of the pair. Furthermore, inspection of standard deviations for data obtained with the instructions to match to only the second stimulus showed a decrease in variability as the time interval was lengthened, suggesting that the task became easier for the longer time intervals.

In our final experiment. instructions to match to the second stimulus were used when the two stimuli in the pair were presented in different channels. Zwislocki and Sokolich (1974) found that when the first stimulus was $4 \mathrm{kHz}$ and when the second and comparison stimuli were $1 \mathrm{kHz}$ there was no enhancement effect, even when the loudness level of the first stimulus was $20 \mathrm{~dB}$ greater than that of the second stimulus. Accordingly, it was predicted that no effect would occur for cutaneous stimuli when the first stimulus was $25 \mathrm{~Hz}$ and the second and comparison stimuli were $300 \mathrm{~Hz}$. As seen in Figure 8, our prediction was clearly confirmed when the first stimulus was increased $10 \mathrm{~dB}$ relative to an equal subjective magnitude match, as well as when the stimuli in the pair had equal sensation magnitudes. The finding is a strong departure from the result of measuring enhancement effects in either mechanoreceptor system alone (Figures 5, 6, and 7). The rather strong enhancement effect produced in both systems when the first burst is $10 \mathrm{~dB}$ above the second disappears when the two systems are made to interact. Our finding is consistent with the data of Zwislocki et al. (1974), in which the enhancement effect disappeared when the frequency of the first burst was outside of the critical band of the second and a maximum effect occurred when the burst pair consisted of nearly equal frequencies.

The effects of interactions between the high- and low-frequency cutaneous receptor systems are summarized in Figure 9. Within either of the two systems, the effect produced by instructions to match to the second stimulus alone (Figure 9a) is strong for short time intervals when the intensity of the first stimulus is raised $10 \mathrm{~dB}$ above the second. The effect diminishes as the time interval is lengthened. Enhancement effects disappear when the first and second stimuli are presented in different systems. Instructions to match to the subjective intensity of the pair produce a 3-dB effect (Figure $9 b$ ) when all frequencies are within one mechanoreceptor system. The summation effect is more pronounced across all time intervals when the frequency of each stimulus activates a different tactile system. The effect produced by the interaction of the two cutaneous

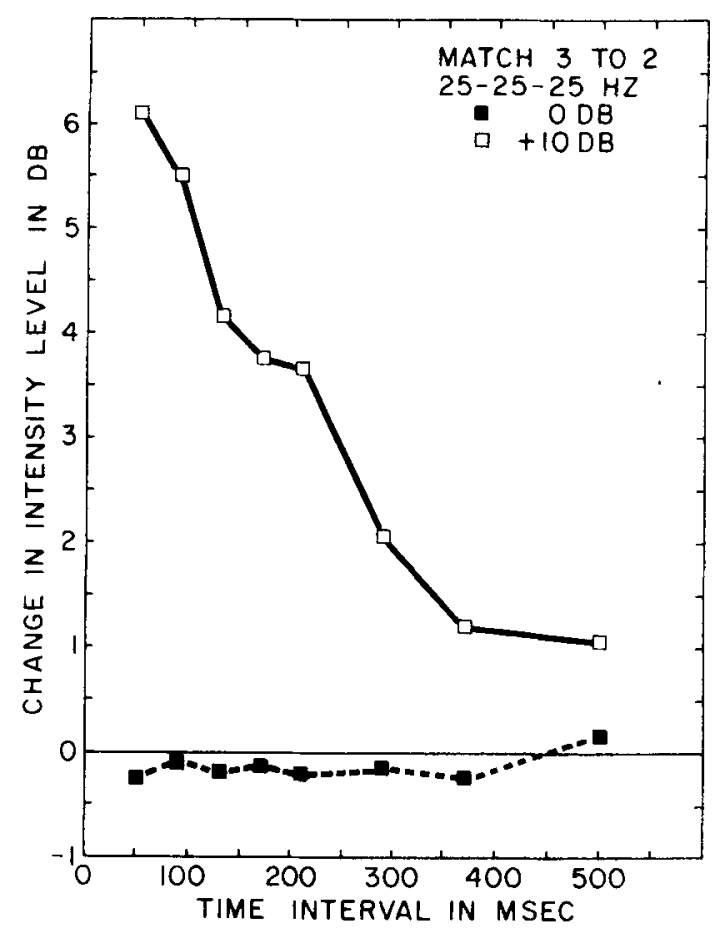

Figure 7. Responses to enhancement instructions plotted as a function of the time interval between the first two stimuli. The stimulus frequencies $(25-25-25 \mathrm{~Hz})$ activate primarily the low-frequency mechanoreceptor system.

mechanoreceptor systems resembles the effect in hearing produced by the interaction among critical bands.

\section{CONCLUSIONS}

The results of our experiments indicate that there are remarkable similarities between auditory and tactile perceptions of pairs of consecutively presented

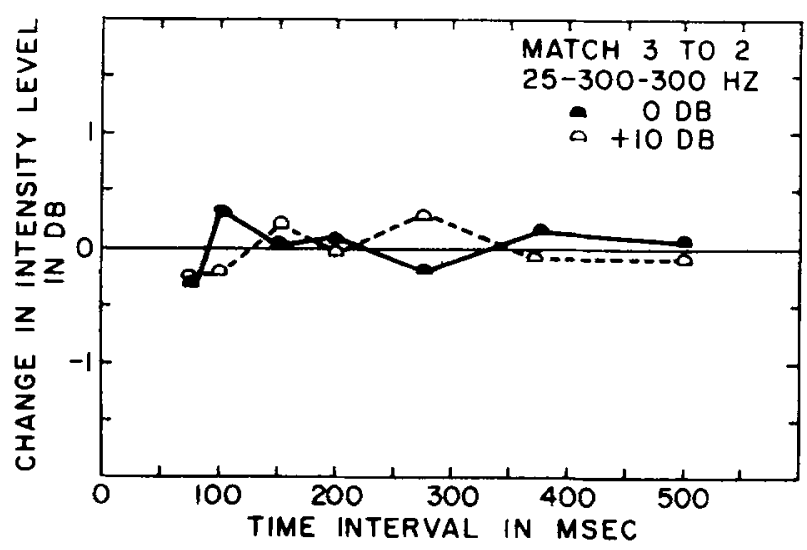

Figure 8. Responses to enhancement instructions plotted as a function of the time interval between the first two stimull. The first stimulus frequency $(25 \mathrm{~Hz})$ is in the low-frequency mechanoreceptor range and the second $(300 \mathrm{~Hz})$ is in the high-frequency range. 


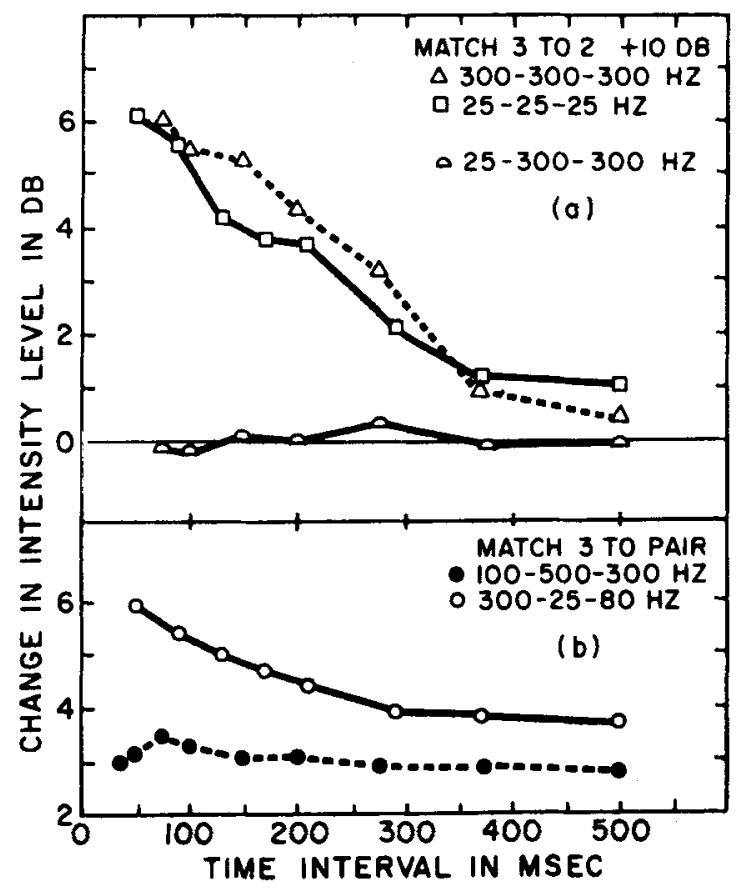

Figure 9. (a) The effects of enhancement instructions when the stimulus frequencies in the pair result in activation of either mechanoreceptor system alone (triangle: $300-300-300 \mathrm{~Hz}$; or square: 25-25-25 Hz), compared with the effects when the frequencies result in activation of both mechanoreceptor systems (half circle: $25-300-300 \mathrm{~Hz}$ ). (b) The effects of summation instructions when the stimulus frequencies in the pair activate only one mechanoreceptor system (closed circles: 100-500-300 $\mathrm{Hz}$ ) compared with the effects when the frequencies result in the activation of both mechanoreceptor systems (open circles: 300-25-80 Hz).

brief stimuli. In both modalities, enhancement and summation were found to be fundamentally different processes which determine sensation magnitude effects for temporally separated stimuli. The enhancement process was found to affect the sensation magnitude of individual stimuli, while the summation process affected the total sensation magnitude of the pair of stimuli. For both auditory and tactile stimuli, the process measured depends on the instructions to the subject and on the frequency of the comparison stimulus relative to the component frequencies of the stimulus pair. The principle of maximum similarity, which refers to the tendency for subjects to match a comparison stimulus to the perceptual aspect of the stimulus pair most resembling the perception of the comparison stimulus, was found to apply to the tactile as well as to the auditory modality.

Another interesting similarity between the auditory and tactile modalities was found when the frequency difference between the first and second stimulus of the pair was manipulated. For both modalities, the enhancement effects were maximum when the frequencies of the two stimuli were identical, but the summation effects were maximum when the frequencies were greatly different. This finding suggests that presenting two tactile stimuli to different receptor populations may be analogous to presenting auditory stimuli in two widely separated critical bands, whereas presenting tactile stimuli to the same receptor population may be analogous to presenting auditory stimuli within the same critical band. Further experimentation is necessary to determine the limits of the auditory critical band analogy for the perception of tactile vibration of variable frequency.

\section{REFERENCES}

CANnon, M. W., \& ZwisLocki, J. J. Loudness summation of pairs of short sounds. Journal of the Acoustical Society of America, $1970,48, a)$.

Galambos, R., Bauer, J., Picton, T., Squires, K., \& Squires, N. Loudness enhancement following contralateral stimulation. Journal of the Acoustical Society of America, 1972, 52, $1127-1130$.

Hunt, C. C. On the nature of vibration receptors in the hind limb of the cat. Journal of Physiology (London), 1961, 155, 175-186.

IRWIN, R. J., \& ZwISLOCKI, J. J. Loudness effects in pairs of tone bursts. Perception \& Psychophysics, 1971, 10, 189-192.

Loewenstein, W. R., \& Mendelson, M. Components of receptor adaptation in a Pacinian corpuscle. Journal of Physiology (London), 1965, 177, 377-397.

SAro, M. Response of Pacinian corpuscles to sinusoidal vibration. Journal of Physiology (London), 1961, 159, 391-408.

SCHARF, B. Dichotic summation of loudness. Journal of the Acoustical Society of America, 1969, 45, 1193-1205.

SChARF, B. Loudness and frequency selectivity at short durations. In R. Plomp \& G. F. Smoorenburg (Eds.), Frequency analysis and periodicity detection in hearing. Leiden: Sijthoff, 1970.

STEVENS, S. S. The measurement of loudness. Journal of the Acoustical Society of America, 1955, 27, 815-829.

Stevens, S. S. Tactile vibration: Dynamics of sensory intensity. Journal of Experimental Psychology, 1959, 57, 210-218.

Talbot, W. H., Darian-Smith, I., Kornhuber, H. H., \& Mountcastle, V. B. The sense of flutter-vibration: Comparison of the human capacity with response patterns of mechanoreceptive afferents in the monkey hand. Journal of Neurophysiology, 1968, 31, 301-334.

Verrillo, R. T. Temporal summation and vibrotactile sensitivity. Joumal of the Acoustical Society of America, 1965, 37, 843-846.

VerRILLo, R. T. Vibrotactile sensitivity and the frequency response of the Pacinian corpuscle. Psychonomic Science, 1966, 4, 135-136. (a)

Verrillo, R. T. Specificity of a cutaneous receptor. Perception \& Psychophysics, 1966, 1, 149-153. (b)

VERRILLO, R. T. A duplex mechanism of mechanoreception. In D. R. Kenshalo (Ed.), The skin senses. Springfield, Ill: Thomas, 1968.

Verrillo, R. T., Fraiol, A. J., \& Smith, R. L. Sensory magnitude of vibrotactile stimuli. Perception \& Psychophysics, $1969,6,366-372$.

Zwicker, E., Flottorp, G., \& Stevens, S. S. Critical band width in loudness summation. Journal of the Acoustical Society of America, 1957, 29, 548-557. 
ZWICKER, E., \& SCHARF, B. A model of loudness summation. Psychological Review', 1965, 72, 3-26.

ZWISLOCKI. J. Theory of temporal auditory summation. Journal of the Acoustical Society of A merica. 1960, 32, 1046-1060.

ZwislockI, J. J., \& KETKAR, I. Loudness enhancement and summation in pairs of short sound bursts. Journal of the Acoustical Society of America, 1972, 51, 140(A).

Zwislocki, J. J., KetKar, I., Cannon, M. W., \& Nodar, R. H.
Loudness enhancement and summation in pairs of short sound bursts. Perception \& Psychophysics. 1974, 16, 91-100.

ZWISLOCKI, J. J., \& Sokolich, W. G. On loudness enhancement of a tone burst by a preceding tone burst. Perception \& Psychophysics, 1974, 16, 87-90.

(Received for publication January 13. 1975 ; revision accepted April 25, 1975.) 